\title{
EDUCACIÓN Y ATENCÓN A LA PRIMERA INFANCIA: CONCEPCIÓN Y SÍNTESIS HISTÓRICA
}

\section{EDUCATION AND EARLY CHILDHOOD CARE: CONCEPTION AND HISTORICAL SYNTHESIS}

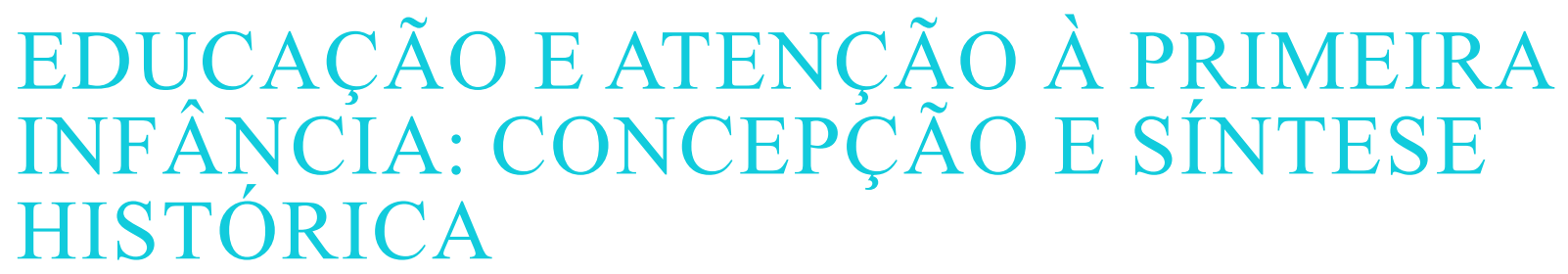

\section{Catalina Trujillo Vanegas ${ }^{1}$}

\section{Willian Sierra Barón²}

\section{Julieth Milena Rincón Perdomo ${ }^{3}$}

Universidad Surcolombiana, Neiva- Colombia

$1 \quad$ catalina.trujillo@usco.edu.co Catalina Trujillo Vanegas. Licenciada en Educación Preescolar, Especialista en Comunicación y Creatividad para la Docencia y Magíster en Educación de la Universidad Surcolombiana (Neiva-Colombia). Candidata a Doctora en Educación y Cultura Ambiental de la Universidad Surcolombiana.

-ORCID: https://orcid.org/0000-0003-2214-7487

$2 \quad$ willian.sierra@usco.edu.co Willian Sierra Barón. Psicólogo y Magíster en Educación de la Universidad Surcolombiana (Neiva-Colombia). Especialista en Gerencia de la Salud Ocupacional de la Fundación Universitaria María Cano (Medellín-Colombia) y Especialista en Gerencia de los Recursos Humanos del Real Centro Universitario Escorial María Cristina (Madrid-España). Candidato a Doctor en Psicología de la Universidad Católica de Colombia. -ORCID: https://orcid.org/0000-0002-7642-477X 3 milena841@hotmail.com Julieth Milena Rincón Perdomo. Licenciada en Pedagogía Infantil, Especialista en Integración Educativa para la Discapacidad y Magíster en Educación de la Universidad Surcolombiana (NeivaColombia). Doctoranda en Bioética de la Universidad El Bosque (Bogotá-Colombia).

-ORCID: https://orcid.org/0000-0002-7665-2705

\section{RESUMEN}

El presente trabajo tuvo como objetivo documentar elementos que han definido el marco de referencia de la educación y la primera infancia, así como aportar de forma concreta algunos elementos históricos que han caracterizado la atención a la primera infancia y distintos procesos educativos. Se esbozan algunas concepciones que han orientado la atención a la primera infancia, en un recorrido histórico por perspectivas en Europa, Estados Unidos, Latinoamérica y Colombia. Finalmente, se hace énfasis en el horizonte y sentido de la atención a la primera infancia en el contexto 
nacional. El texto concluye que los desafíos que se presentan en la atención integral a la primera infancia han sido objeto de reestructuración constante a través de las politicas que las han definido y que se han orientado hacia la educacion, el cuidado y la proteccion de los menores, guiado principalmente por variaciones en la evolucion sobre el concepto y comprension de la infancia.

PALABRAS CLAVE: primera infancia, atención integral, educación, políticas públicas, historia, equidad, colombia.

\section{ABSTRACT}

The purpose of this work was to document elements that have defined the frame of reference for education and early childhood, as well as to provide in a concrete way some historical elements that have characterized early childhood care and different educational processes. Some conceptions that have oriented attention to early childhood are outlined in a historical journey through perspectives in Europe, the United States, Latin America and Colombia. Finally, emphasis is placed on the horizon and sense of early childhood care in the national context. The text concludes that the challenges presented in comprehensive early childhood care have been subject to constant restructuring through the policies that have defined them and that have been oriented towards the education, care and protection of minors, guided mainly by variations in the evolution of the concept and understanding of childhood.

KEYWORDS: early childhood, comprehensive care, education, public policies, history, equity, Colombia.

\section{RESUMO}

O objetivo deste trabalho foi documentar elementos que definiram o quadro de referência para a educação e a primeira infância, bem como fornecer de maneira concreta alguns elementos históricos que caracterizaram os cuidados na primeira infância e os diferentes processos educacionais. Algumas concepções que orientaram a atenção para a primeira infância são descritas em uma jornada histórica através de perspectivas na Europa, Estados Unidos, América Latina e Colômbia. Finalmente, a ênfase é colocada no horizonte e no sentido dos cuidados na primeira infância no contexto nacional. $O$ texto conclui que os desafios apresentados nos cuidados abrangentes da primeira infância foram sujeitos a constante reestruturação por meio das políticas que os definiram e que foram orientadas para a educação, o cuidado e a proteção dos menores, guiados principalmente por variações na evolução do conceito e entendimento da infância.

PALAVRAS-CHAVE: primeira infância, atenção integral, educação, políticas públicas, história, equidade, Colômbia.

\section{INTRODUCCIÓN}

Los desafíos que hoy persisten en la atención integral a la primera infancia se ven íntimamente relacionados con la eficacia en la garantía de los derechos de esta población, para lo cual los estados y sus instituciones se han visto abocados a repensar y reestructurar permanentemente sus políticas y programas orientados para la educación, protección y cuidado de los niños y niñas más pequeños (Ardila, Mantilla \& Bolivia, 2013; Bácares, 2014; Myers, 2000). Esta tendencia llama la atención de toda la sociedad en cabeza de las familias en proveer de condiciones adecuadas para que el desarrollo de los pequeños, esté articulada no solo a sus necesidades, sino a sus aspiraciones y nociones del mundo, para lo cual el papel del entorno resulta fundamental en cuanto a la reciprocidad y cambios permanentes en el que 
los niños se ven involucrados, pero a la vez en el que ellos mismos pueden incidir (Rincón, 2019; Rozo-Gutiérrez \& Vargas-Trujillo, 2018; Sánchez, Ofir, Trujillo \& Pérez, 2016).

Desde el Modelo Ecológico (Bronfenbrenner,1987), la evolución del niño se entiende como un proceso de diferenciación progresiva de las actividades que éste realiza, de su rol y de las interacciones que mantiene con el ambiente. Se resalta la importancia de las interacciones y transacciones que se establecen entre el niño y los elementos de su entorno, empezando por los padres y los iguales (Bronfenbrenner,1994; Bronfenbrenner,1987). De acuerdo con estas ideas, al analizar el desarrollo del niño, no se debería considerar sólo su comportamiento de forma aislada, o como fruto exclusivo de su maduración, sino siempre en relación con el ambiente en el que se desarrolla (Abeya et al., 2004; Viciana, Cano, Chacón, Padial \& Martínez, 2017; Vigotsky, 1979).

\section{¿Cuál ha sido la concepción de la Atención a la primera infancia a través del tiempo?}

Resulta una premisa aceptada aquella que establece que el crecimiento de los niños y las niñas va de la mano de los esfuerzos de las sociedades por pensar una educación acorde a sus necesidades, que articula el desarrollo físico - cognitivo con el social y emocional, y a la vez que guía los pasos de los diferentes actores corresponsables en la formación holística e incluyente de estos (Guedeney \& Pérez, 2015; Pineda \& Orozco, 2018; Rodríguez, 2000). Desde el objetivo de la Educación para todos del Marco de Acción de Dakar (Unesco, 2000), se estableció una perspectiva de lo que debía ser la atención y educación a la primera infancia:

La atención y educación de la Primera Infancia sustentan la supervivencia, el crecimiento, el desarrollo y el aprendizaje de los niños -incluyendo aspectos como salud, nutrición, higiene y el desarrollo cognitivo, social, físico y afectivo- desde su nacimiento hasta su ingreso en la escuela primaria en contextos formales, no formales e informales (Ancheta, 2010, p.2).

Para dar claridad a esta premisa resulta importante revisar la evolución del concepto de infancia que constituye hoy la justificación de lo que se determina es la atención y educación para esta población. En los siglos XV y XVI se concebía al niño como indefenso, como algo inacabado, una propiedad particular del adulto (Jaramillo, 2007). Para el siglo XVII se le dota al infante de una "bondad innata" casi espiritual y por tanto ajena a la realidad terrenal. En el siglo XVIII persiste la idea del niño como algo que le falta ser, un ser primitivo. Es a partir del siglo $X X$ hasta la actualidad, que se concibe al infante como un sujeto social de derecho gracias a las reivindicaciones sociales e investigaciones académicas defensoras de los niños y las niñas (Jaramillo, 2007).

Dentro de las subcategorizaciones asignadas a la niñez se encuentran las etapas o ciclos de la infancia. Una de ellas, la primera infancia, la cual se comprende como la etapa del desarrollo que incluye desde la gestación hasta la edad de 7 años, que está caracterizada por rápidos cambios que se producen (Jaramillo, 2007).

Entre los múltiples conceptos asociados y construidos sobre la infancia, se encuentra aquellos alternativos y controversiales que sitúa al menor como actor con niveles importantes de autonomía, resultado de las transformaciones de la familia, los desarrollos socioeconómicos de las sociedades y las diferencias generacionales que chocan con la idea de la sumisión y control de los infantes; es decir, que son sujetos a los que hay que socializar y educar, para lo cual se estima que por el contrario éstos son "actores sociales competentes que participan en el moldeado de sus ambientes" (Ancheta, 2013, p.4). 
A partir del siglo XXI la idea que prevalece es la del niño como sujeto social de derecho, tal como lo planteó la UNESCO (1996) en la Comisión Internacional sobre la Educación para el siglo XXI, donde estipulo la formación integral del niño a partir de las dimensiones del aprender a conocer, aprender a hacer, aprender a ser y aprender a vivir juntos (Delors, 1996). Lo anterior se da con el precedente de la Convención Internacional de los derechos del Niño (UNICEF, 2006), que lo reconoce como persona y ciudadano. Concebir al infante como sujeto de derechos orientó a los Estados a pensar en el menor como objeto de políticas sobre el cual debía recaer y prevaler leyes conducentes al reconocimiento de este nuevo grupo poblacional, y velar tanto por su bienestar como por su dignidad.

Las instituciones que representan por excelencia la idea de la atención a la infancia son las escuelas, de allí surgen otras miradas y concepciones necesarias sobre la infancia como sucede desde la comprensión pedagógica moderna, en el que la infancia se entiende como un periodo de desarrollo y transición a la vida adulta y desde la concepción contemporánea se comprende esta etapa como un periodo de la vida de desarrollo psicobiológico y social que se da desde los procesos educativos institucionales (Alzate, 2002).

Por otro lado, son nacientes las perspectivas que señalan la labor de las instituciones del estado como un proveedor de soluciones parciales a la realidad y problemáticas que aquejan a la infancia, pues, su papel limitado frente a la esfera privada de la familia, lo ha dejado asumiendo políticas de bienestar vinculadas principalmente a la educación, por lo tanto se hace necesario transitar de la comprensión de la infancia vista desde las necesidades que emergen a una infancia desde el enfoque de derechos, que desde la praxis no se afecte los recursos y potencialidades de los niños y niñas desde su gestación (SENSAT, 2004).

\section{Recorrido histórico en Europa y Estados Unidos}

La idea moderna de la educación para los niños, fue dada por Jean-Jacques Rousseau en el siglo XVIII, quien desde la concepción del niño como un ser bueno por naturaleza, pero expuesto a la perversión de la sociedad, insiste en la necesidad de una educación que se adapte al menor y exalte la acción y la experiencia como oportunidades importantes para adquirir conocimiento, más allá de la memorística, superando con ello la noción medieval del niño como homúnculo (hombre en miniatura) que no evoluciona y que por tanto es defectuoso (Figari, 2009; Vilafranca, 2012).

Como tal la Educación y Atención a la Primera Infancia (EAPI) tiene antecedentes no muy remotos en el siglo XIX, para el caso de los Estados-Nación se establecieron escuelas infantiles para párvulos, en países como Suecia y Francia entre los años 1836 y 1881 (Bennett, 2011). Los propósitos de las dos instituciones fueron muy diferentes debido a que aquellas orientadas al cuidado infantil se enfocaron en la supervivencia y la protección a nivel social, y por otro lado las escuelas evidenciaron un interés más educativo, por lo tanto se instituyeron los jardines infantiles y la escuela preescolar para brindar así una coyuntura en la educación para niños de mejores condiciones sociales para lograr la inserción de la lengua y la cultura oficial a los niños que hablaban un segundo idioma (Bennett, 2011, p.1)

Ya para el siglo $\mathrm{XX}$, las iniciativas del movimiento de la caridad privada, $y$ en las políticas subsiguientes a la II guerra mundial dieron surgimiento a las políticas públicas de cuidado a los niños más pobres y desprotegidos de la sociedad; a esto se sumó las aceleradas transformaciones de los países occidentales, 
que tras los procesos de globalización, neoliberalismo y mercantilismo, reconfiguraron las políticas que dieron origen a los modernos sistemas educativos y sociales para los niños y niñas (Midaglia, Barba \& Lomelí, 2018). Dentro de los factores que han incidido en los cambios de las políticas de educación y atención en primera infancia en los países desarrollados están: la aspiración de aumentar en el ámbito laboral la presencia de las mujeres, la resolución de las problemáticas a nivel demográfico y el análisis de las situaciones como pobreza infantil (Ancheta, 2013).

La EAPI por tanto es considerada por los grupos de países de la Unión Europea como un bien público al que deben disponer todos los recursos y condiciones para su efectivo desarrollo, de manera que pueda verse en la primera infancia la prolongación generacional que garantizará el bienestar social venidero. En este sentido, la primera infancia se constituye como una etapa de inversión para el desarrollo humano (Heckman, 2004).

En los años 80, organismos como el Fondo Monetario Internacional, Naciones Unidas y el Banco Mundial influyeron en las políticas de desarrollo con una marcada tendencia a privilegiar lo privado sobre lo público y lo individual sobre lo colectivo, de manera que ha trasladado la responsabilidad del Estado a la responsabilidad del individuo sobre su propio bienestar, en donde lo fundamental de esta nueva moral económica se orienta hacia la privatización de la responsabilidad (Ancheta, 2013; Narodowski \& Martínez, 2015).

La Organización para la Cooperación y el Desarrollo Económico (OCDE) publicó en el 2006 las directrices que dieron paso a la política pública en educación y cuidado de la primera infancia; de ellas se desprendieron cuarto bloques que orientaron los esfuerzos realizados por estos países para promover el acceso equitativo y de calidad en educación a los niños y niñas ubicados en la etapa de la primera infancia (Ancheta, 2013; SerranoArenas, Ochoa-Cervantes \& Arcos-Miranda, 2019), a saber:

i) El enfoque sistemático e integrado sobre el desarrollo y puesta en práctica de políticas de educación y atención para la primera infancia. Coherente con el sistema educativo apoyándose en el aprendizaje para toda la vida y que persiga la universalidad, con especial énfasis en niños que requieren atención especial.

ii) Principales inversiones públicas en servicios e infraestructuras.

iii) Modelo participativo para mejorar y asegurar la calidad. Formación y condiciones de trabajo idóneas para el personal de educación y atención preescolar.

iv) La sistematización de la recogida de datos y seguimiento. Investigación y evaluación en un marco estable y un programa a largo plazo.

Hoy en los países europeos la atención está puesta sobre la eficiencia, calidad, equidad e inclusión en la educación de los más pequeños, trasversalizada por la garantía de sus derechos, que advierten sobre las necesidades especiales atribuidas a la crianza, protección y educación (Ancheta, 2013; Ducoing \& Barrón, 2017). En este, la educación en la primera infancia considera dos elementos de importancia: el aprendizaje y la atención y educación; en el primer caso se reconoce como un proceso que se desarrolla desde el nacimiento. Para el caso de la atención y educación como tópicos que no deben tratarse de forma independiente (ZapataOspina \& Restrepo-Mesa, 2013).

Para el caso de los Estados Unidos, la atención y educación de la primera infancia guarda sus principales antecedentes en la iniciación de la época moderna (Siglo XX), para lo cual se 
exaltan dos programas de política de atención a la primera infancia: "Head Start" y "Cuidado del niño". El objetivo del primer programa era preparar a los niños pobres para ingresar en la escuela de la mano con los servicios en salud, educación y familiar, mientras que el segundo programa tenía como propósito favorecer las condiciones de trabajo para que los padres tuvieran económicamente como aportar al cuidado de los niños (Lombardi, 2004; Quintero, Ramírez \& Jaramillo, 2016).

Progresivamente se fue trasladando el enfoque asistencial a uno más integral que pensará en la atención y educación no como estrategias separadas, sino como un binomio que garantizará el acceso equitativo a los servicios prestados por el Estado, ya no era solo iniciativa de cada federación en pensar en estrategias locales para la población infantil, sino que se transitó en una perspectiva más universalista en donde se privilegiaba a los niños y niñas con necesidades particulares. De esta manera se fue aumentando la fracción población de la primera infancia de 0-2 años, luego de 0-5 y en la actualidad se contempla como la primera etapa de la infancia la edad de los 0 a 8 años (Bernal \& Camacho, 2012; Bodero, 2017).

En aras de avanzar en la articulación de los diferentes programas para la primera infancia, Estados Unidos le apostó a una mayor coordinación entre la gobernabilidad, estándares, sistemas de desarrollo profesional, recolección de datos, fondos públicos en un sistema de distribución alternativo, sistema de calificación "estrella" - niveles de estándares de calidad, de este modo se estableció el Sistema Estatal de Desarrollo de la Primera Infancia (Lombardi, 2004; Torres \& Ramos, 2016) como se muestra en la siguiente figura:

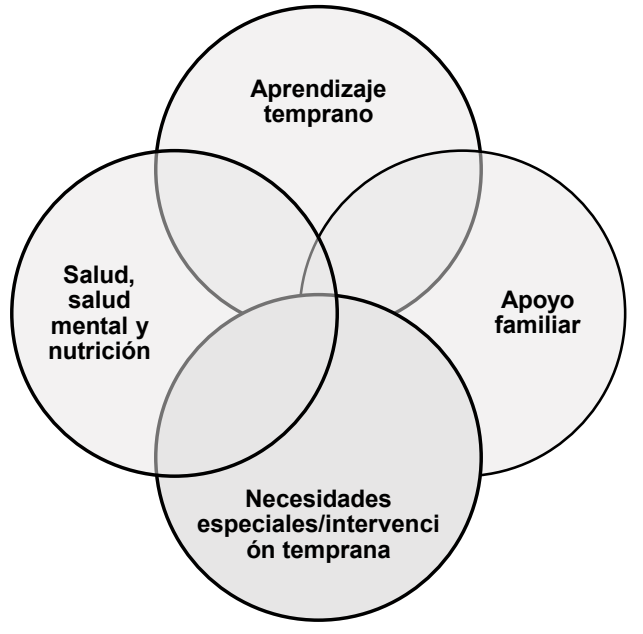

Figura 1. Sistema Estatal de Desarrollo de la primera infancia. Tomado de Lombardi (2004)

\section{Recorrido Latinoamericano}

En América Latina, los programas de atención a la primera infancia datan de hace un siglo y medio, con las experiencias de jardines infantiles que cumplían un papel primordialmente asistencial "Caridad o Beneficencia" al menor. A finales del siglo XX las preocupaciones por la primera infancia, considerada entonces desde el nacimiento hasta los 6 años, tuvieron origen en las dinámicas socioeconómicas y en el impacto del proceso de atención al crecimiento, desarrollo y aprendizaje de los niños. Sumada a esta preocupación se relacionaban la necesidad de mejorar las condiciones de ingreso de los niños a la educación básica así mismo como mejorar las condiciones de trabajo fuera del hogar para la mujer y la calidad de vida de las comunidades (Peralta \& Fujimoto, 1998; Rodríguez \& Marzonetto, 2015).

Las dimensiones atendidas inicialmente a la primera infancia fueron las de salud, protección y educación. El ámbito de la salud fue priorizado debido a los altos índices de mortalidad y morbilidad infantil debido a los volúmenes tan altos de pobreza en las principales ciudades latinoamericanas, lo que llevo a generar 
programas de higiene, alimentación y cuidado para las madres y los niños. Para el caso de la protección y bienestar del menor, surgieron desde las instituciones programas orientados a salvaguardar a los menores huérfanos, abandonados e indigentes, que antes habían sido solo atendidos por las entidades religiosas; esos nuevos panoramas de desprotección y desamparo del menor propició en las naciones leyes y códigos de protección al infante (Universidad de Costa Rica, 2015). Finalmente, en el escenario educativo, América Latina fue influenciada por los vientos renovadores de Europa, debido a que el movimiento pedagógico visibilizo los planteamientos de la escuela activa que se daban a partir de las ideas de los representantes teóricos de la época sobre la educación de los niños como Comenio, Pestalozzi, Frobel, Agazi, Montesori, y Decreoly, y que produjo que en América latina se desarrollara preocupación por la educación y particularmente por la educación infantil (Peralta \& Fujimoto, 1998 ). Se estima que dentro de las ideas fundantes de la educación inicial en América Latina se encuentran:

i) El derecho que tiene el párvulo, desde que nace, a una educación permanente $y$, por tanto -oportuna- dentro de un planteamiento de un continuum educacional para el ser humano.

ii) El supremo interés del párvulo, a partir de sus características y necesidades, como eje de una educación pertinente.

iii) La educación del párvulo se plantea como complementaria a la que realiza la familia, por lo que genera acciones conjuntas para el logro de los objetivos que se pretenden en función a los niños.

iv) La educación del párvulo se plantea tempranamente como una propuesta de equidad para los sectores pobres y una solución para la madre que trabaja (Cordero, 2004; Ruiz, 2013).

En América latina se han realizado avances en políticas del cuidado y educación para los niños y las niñas; sin embargo, se presenta como reto llegar a las poblaciones vulnerables desde lo inclusivo junto con políticas orientadas hacia la superación de la condición de pobreza (Paya, 2010; Vaillant, 2019; UNESCO, 2007), ya que el centro de la problemática real de beneficio y garantía de los derechos de los niños está situado en el panorama de desigualdad.

Sin lugar a duda los avances en políticas para la educación y atención a la primera infancia ha tenido repunte en el actual siglo, donde se ubican niveles importantes de priorización (social, política y presupuestal), articulación interinstitucional, mayor cualificación de los servidores responsable de la EAPI, apertura de estudios sobre el área, vinculación y actualización de sistemas de seguimiento que ofrecen las nuevas tecnologías de la información y la comunicación. Pero así mismo se reconoce los desafíos y limitaciones que persisten no solo en las políticas para la primera infancia, sino en el contexto sujeto al desarrollo de los niños y las niñas, como es el caso de la deficiente información estadística, de la necesidad de ganar mayor coherencia entre la definición política y financiera dirigida a los programas para los niños y niñas más pequeños, como en establecer una clara definición de los roles del Estado y la sociedad civil que adelantan programas de EAPI, y finalmente dirigir mayores esfuerzos en garantizar calidad, equidad e inclusión en los programas que benefician a los niños (Azorín, 2017; Díaz, Arancibia, Bello, Salinas \& Sánchez, 2016; Tuñón, 2015).

Hoy se destaca la perspectiva de la Teoría de los Sistemas (Cathalifaud \& Osorio, 1998) para evaluar el desarrollo infantil, que había estado orientado desde la nueva Teoría Institucional y la 
Teoría Adaptativa compleja. Mientras la primera le puso acento a la prestación de los servicios de las instituciones donde se analiza la cultura normativa y la influencia del contexto en el comportamiento institucional y organizacional. La Teoría Adaptativa (García, 1997) partió de las condiciones complejas de la sociedad contemporánea y advirtió que los cambios son normativos y no lineales, dado que no se puede analizar una sola institución o programa sino todos en su conjunto de una forma sistémica. Finalmente, la Teoría de los Sistemas permite complementar y comprender las acciones de la infancia temprana y que aporta a las teorías contemporáneas sobre el desarrollo (Lynn, Caridad, Jamovich \& Cruz-Aguayo, 2016).

\section{COLOMBIA FRENTE A LA PRIMERA INFANCIA}

En Colombia la entrada en vigor de los estudios y temáticas en relación con la primera infancia tiene sus inicios en el siglo $X X$, en una idea más cercana a los patrones de crianza y amamantamiento; luego a los patrones de educación, de relación familiar y maltrato que fueron tratados por las instituciones religiosas de acuerdo con los vestigios de la colonia (Izzedin \& Pachajoa, 2009).

Bajo la misma corriente Europea y Latinoamericana, la niñez en Colombia transitó por la invisibilización de la época de herencia medieval, como por la contradicción del entendimiento de la figura del infante como bueno pero pervertible, hasta llegar a reconocerlo como sujeto social con igualdad de derechos (Adams 2010; Pachón, 2007).

Frente a esta etapa de la vida en concreto, que va desde los 0 años (desde la gestación) hasta los 6 años, el Código de Infancia y Adolescencia (Ley 1098, 2006) la ha descrito en su artículo 293 como un periodo de la vida en el que se generan las bases para lograr el desarrollo a nivel cognitivo, emocional y social.

Ante esa descripción se establecieron 4 grupos de derechos sobre los cuales primaría la atención a la infancia, estos fueron: Existencia (Todos nacidos vivos, todos saludables, ninguno sin familia y ninguno desnutrido), Desarrollo (Todos con educación, todos jugando y todos capaces de manejar los afectos, las emociones y la sexualidad), Ciudadanía (Todos registrados) y Protección (Todos protegidos ante los perjuicios a la integridad humana) (UNICEF, 2005).

Luego del convenio con los acuerdos internacionales para el debido cumplimiento de los derechos de los menores, que implica desde el nacimiento hasta la edad de 6 años, en el territorio Colombiano se propendieron por actividades como "la movilización por la primera infancia que tuvo sus orígenes en el 2002, en la Alianza por la Política Pública de Infancia y Adolescencia en Colombia, a través de un grupo de trabajo integrado por el Instituto Colombiano de Bienestar Familiar ICBF, el Departamento Administrativo de Bienestar Social DABS, el Centro Internacional de Educación y Desarrollo -y el Fondo de las Naciones Unidas para la Infancia. ICBF" (Instituto Colombiano de Bienestar Familiar, 2006, p. 3)

\section{COMPRENSIÓN DE LA ATENCIÓN INTEGRAL A LA PRIMERA INFANCIA COLOMBIANA}

Como experiencias vivenciales e institucionales de atención integral a la primera infancia se destacan en los años $70^{\circ}$ los Hogares Infantiles (antiguos Centros de Apoyo Integral Pedagógico - CAIPS), ya para el $86^{\circ}$ se adopta el programa de Hogares Comunitarios de Bienestar, y en los $90^{\circ}$ en cabeza del ICBF Colombia empezó a orientar la atención integral de la primera infancia de acuerdo a prácticas pedagógicas guiadas 
por esta institución a partir de tres documentos rectores: "El Proyecto Pedagógico Educativo Comunitario en el ICBF (1990), El Desarrollo Infantil, una conceptualización desde el ICBF (1990) y La Escala de Valoración Cualitativa del Desarrollo Infantil, desde el nacimiento hasta los seis años- manual (1995)" (ICBF, 2006,p.26). Para 1996 se incorpora una nueva estrategia de promoción del desarrollo infantil, orientada a las madres gestantes o con hijos menores de dos años: el programa Familia, Mujer e Infancia -FAMI-(Arboleda \& Sierra, 2008).

De igual forma, se gestaron una serie de leyes, acuerdos y programas conducentes a la atención integral de la primera infancia, dentro de los que se destacan:

i) La construcción participativa de política pública de infancia denominada Colombia por la Primera Infancia que es una política pública por los niños y niñas desde la gestación hasta los 6 años (ICBF, 2006).

ii) Se promulgó, el código de la Infancia y la Adolescencia que deroga el Código del Menor. Esta ley establece en su Artículo 29 el derecho al desarrollo integral de la primera infancia (Ley 1098, 2006).

iii) Se dio aprobación al CONPES 109 (2007), el cual materializa el documento "Colombia por la Primera Infancia" y fija estrategias, metas y recursos al Ministerio de la Protección Social, Ministerio de Educación y al ICBF, con el fin de garantizar la atención integral a esta población.

iv) Se promulgó la Ley 1295 (2009) o de atención integral a la primera infancia, por la cual se reglamente la atención de los niños y niñas de la primera infancia de los sectores 1,2 y 3 de Sisbén, con la que el Estado plantea contribuir a la calidad de vida de las madres gestantes y a garantizar los derechos de las niñas y los niños desde su gestión.

v) De fijaron recursos para la Atención Integral de la Primera Infancia: CONPES 162.

vi) Se estableció el Decreto 4875(2011) por el cual se crea la Comisión Intersectorial para la Atención Integral de la Primera Infancia.

vii) Se publicó el documento Fundamentos Políticos, Técnicos y de Gestión de la estrategia de atención integral a la primera infancia (Alarcón et al.,2013).

Hoy diferentes ámbitos constituyen la significación de la atención integral de la primera infancia. De un lado aparece la concepción científica que refiere que en el cerebro en los primeros años de existencia se desarrolla de tal forma que no vuelve a repetirse, dado que en los primeros tres años de vida se generan de 700 a 1000 conexiones cerebrales por segundo, estas conexiones dependen de las relaciones interpersonales que los niños y niñas establecen con sus cuidadores y de los estímulos tanto positivos como negativos que se presenten en el medio que interactúan (Fondo de las Naciones Unidas para la Infancia, 2014).

Así mismo, social y culturalmente es necesario llamar la atención sobre la concepción del desarrollo humano como un proceso multidimensional que demanda un conjunto de condiciones que deben ser garantizadas, tales como la salud, la nutrición, la educación, el desarrollo social y el desarrollo económico. Esta concepción conlleva a la redefinición de la atención a la primera infancia en términos de integralidad, proceso en el que tiene especial relevancia la educación inicial como proceso social que genera impactos positivos sobre el desempeño escolar y académico de las niñas y niños, su desarrollo humano y económico en la sociedad (ICBF, 2018). 
Entendiéndose por Atención Integral a la Primera Infancia, las acciones que se planifican a nivel nacional y territorial que están orientadas a promover y garantizar el desarrollo de los niños y niñas desde su nacimiento hasta la edad de seis años, este es un trabajo de carácter intersectorial desde los derechos humanos que une y promueve el diseño de planes, programas y acciones que dirigidas a la atención integral de los niños y niñas de acuerdo con su edad, condición y en el contexto en el que se desarrolla (Alarcón et al., 2013).

\section{HORIZONTE Y SENTIDO DE LA ATENCIÓN INTEGRAL A LA PRIMERA INFANCIA}

La estrategia de atención a la primera infancia de Cero a Siempre contempla varios momentos y aspectos necesarios para orientar la garantía de los derechos de los niños y niñas menores de 6 años, para los cuales tanto el Estado como la familia y sociedad cooperan para el real beneficio de la política intersectorial de atención integral al infante (Alarcón, Castro, Schmidt, Turriago \& Carvajal, 2013).

De ese modo, la estrategia ha definido unas condiciones especiales denominadas como realizaciones, que cuentan con atenciones integrales reconocidas como estructurantes, y unas atenciones efectivas por edad denominadas atenciones, que al final se ven trasversalizadas por los entornos asumidos como los escenarios en donde se desarrollan los pequeños como se muestra en la siguiente tabla: 
Tabla 1.

Acuerdos conceptuales - Estrategia de atención integral a la primera infancia.

\begin{tabular}{|c|c|c|}
\hline Elementos & En que consiste & Cuales son \\
\hline $\begin{array}{l}\text { 1.Las } \\
\text { realizaciones }\end{array}$ & $\begin{array}{l}\text { Condiciones y estados que se materializan } \\
\text { en la vida de cada niña y cada niño, y que } \\
\text { hacen posible su desarrollo integral. } \\
\text { Son logros que permiten evidenciar que } \\
\text { realmente se está dando el ejercicio efectivo } \\
\text { de los derechos planeados desde } \\
\text { la "Estrategia de Atención Integral } \\
\text { de Cero a Siempre" en la vida de } \\
\text { los niños y las niñas. }\end{array}$ & $\begin{array}{l}\text { 1. Cuenta con padre, madre o cuidadores principales que la } \\
\text { acogen y ponen en práctica pautas de crianza que favorecen } \\
\text { su desarrollo integral. } \\
\text { 2. Vive y disfruta del nivel más alto posible de salud. } \\
\text { 3. Goza y mantiene un estado nutricional adecuado. } \\
\text { 4. Crece en entornos que favorecen su desarrollo. } \\
\text { 5. Construye su identidad en un marco de diversidad. } \\
\text { 6. Expresa, sentimientos, ideas y opiniones en sus entornos } \\
\text { cotidianos y estos son tenidos en cuenta. } \\
\text { 7. Crece en entornos que promocionan sus derechos y } \\
\text { actúan ante a exposición a situaciones de riesgo o } \\
\text { vulneración }\end{array}$ \\
\hline 2.Los estructurantes & $\begin{array}{l}\text { Acciones que conforma la atención } \\
\text { integral y que debe ser garantizado a } \\
\text { cada niña y a cada niño para asegurar } \\
\text { desarrollo integral. }\end{array}$ & $\begin{array}{l}\text { El cuidado y la crianza } \\
\text { La salud, la alimentación y la nutrición } \\
\text { La educación inicial } \\
\text { La recreación } \\
\text { El ejercicio de la ciudadanía y la participación }\end{array}$ \\
\hline
\end{tabular}

Acciones intencionadas y efectivas encaminadas a asegurar en cada uno de los entornos, existan las condiciones

3. Las atenciones humanas, sociales, y materiales para garantizar la promoción y potenciación de su desarrollo. Varían conforme el momento o edad particular por la que cada sujeto de atención atraviesa.
En edad fértil (preconcepción)

Durante la gestación

En el nacimiento y durante el primer mes de vida

Del primer mes a los tres años

De los tres a los seis años

Son espacios físicos, sociales y culturales donde habitan los seres humanos, en los que se produce una intensa organizativa.

Fuente: Caja de herramientas para la atención integral en salud de niñas, niños y adolescentes (2019). 
La educación inicial garantiza al niño y la niña la inserción adecuada al mundo social, para lo que resulta fundamental que los actores involucrados que están al servicio de este proceso estén acordes a las necesidades y retos que esta delegación de la sociedad tiene sobre cada uno de los pilares constitutivos de la formación y el desarrollo de los pequeños (Blanco, 2005; Salinas-Quiroz et al., 2015).

En este sentido, los procesos de potenciación, estructura y dotación de los niños se nutren de las experiencias que tiene con el medio y los adultos que habitan en él. Dicha interacción se establece en diferentes ámbitos o entornos tanto físicos como simbólicos, tales como: el hogar, el educativo, la salud, el espacio público, entre otros, donde prima el espacio de iniciación del menor, así en el entorno hogar se generan los procesos de crianza y se conforman los procesos que se desarrollan más adelante en la escuela y que permiten que el desarrollo de la identidad, el reconocimiento del otro y el desarrollo de la autonomía (Cárdenas \& Gómez, 2014 ).

La educación inicial es igualmente asumida como parte esencial de la atención integral a la primera infancia. Entonces, el cuidado y la crianza son elementos que hacen parte de la atención integral que sucede en los entornos familiares y educativos (Cárdenas \& Gómez, 2014).

En la política de primera infancia se plantea la importancia de la atención integral para el desarrollo infantil, así como las estrategias que se diseñaron para promover el acceso a este nivel educativo. En ese sentido, se propusieron tres modalidades de atención que se definieron y desarrollaron de acuerdo con la guía 35: Guía operativa para la prestación del servicio de atención integral a la primera infancia (Ministerio de Educación Nacional, 2010), a saber:
Modalidad entorno familiar. Buscaba atención integral en cuidado, nutrición y educación inicial a niñas y niños menores de 5 años ubicados en zonas rurales o urbanas, atendidos y educados por sus familias, fortaleciendo la labor educativa que se brinda en el hogar.

Modalidad entorno comunitario. Pretendía brindar atención integral a las niñas y los niños menores de 5 años acogidos actualmente por los hogares comunitarios del Instituto Colombiano de Bienestar Familiar, como complemento a los servicios de cuidado y nutrición con un componente educativo.

Modalidad entorno institucional. Estaba dirigida a niñas y niños ubicados en zonas rurales y urbanas atendidos por instituciones especializadas en atención integral, donde se ofrecen los componentes de cuidado, nutrición y educación inicial durante 5 días de la semana, en jornadas de 8 horas diarias (Cárdenas \& Gómez, 2014p.35).

Frente a los componentes de servicio y condiciones de calidad para las modalidades, el ICBF hace especial énfasis en organizar los servicios de educación inicial enfocados al desarrollo integral de los niños y las niñas de la primera infancia, de acuerdo con lo estipulado por los programas DIMF y FAMI. De este modo se contemplan 5 componentes (ICBF, 2017):

i) Componente familia, comunidad $y$ redes. Para este caso el rol de la familia se ve abocado no solo al vínculo sanguíneo, sino a la concepción dispuesta por el consejo de Estado que define...entre otras...que "las familias son una unidad en razón a sus vínculos de afecto y a la convivencia, son agentes corresponsables de los derechos de sus integrantes y mediadoras con el Estado y la sociedad para su garantía".

ii) Componente salud y nutrición. Los niños, niñas y madres gestantes cuentan con 
acceso a los servicios de salud, como los "aspectos relacionados con la creación de hábitos saludables y la generación de espacios en condiciones higiénico-sanitarias para la salud de la primera infancia, así como el consumo de los alimentos requeridos de acuerdo con los grupos de edad".

\section{iii) Componente proceso pedagógico.} Tanto el cuidado calificado como el proceso pedagógico se contemplan como dinámicas complementarias que son tenidas en cuenta para favorecer las prácticas pedagógicas que promueven en desarrollo integral de los infantes y sus madres. Para lo cual "la metodología a utilizar para el acercamiento a las familias deberá ser transgeneracional e incluyente de todos los miembros de la familia... indistintamente de su edad, los hacen portadores de saberes y prácticas socioculturales que un proceso educativo requiere reconocer".

iv) Componente talento humano. $\mathrm{Da}$ prioridad a la conformación de equipos multidisciplinarios con destacadas capacidades no solo profesionales, sino vocacionales frente a la atención y servicio de la primera infancia, para lo cual ha sido importante delimitar las áreas de conocimiento desde las diferentes comprensiones de la infancia.

v) Componente Ambientes educativos $y$ protectores. Los espacios en donde se desarrolla la dinámica específica de los servicios de la modalidad familiar son los encuentros grupales y los encuentro en el hogar, lo que ubica la corresponsabilidad del acompañamiento a las familias o cuidadores, que se debe garantizar de manera que se fortalezcan los procesos que van encaminados a configurar los ambientes educativos y protectores de niñas, niños y sus familias" y así potenciar el desarrollo integral.

vi) Componente administrativo y de gestión. Actividades de planeación, organización, ejecución, seguimiento, evaluación y control, dirigidas a alcanzar los objetivos trazados y relacionados con "el desarrollo de políticas sociales, correspondientes con el bienestar de las niñas y niños desde los cero (0) hasta los dos (2) años, o hasta los cinco (5) años cuando aplique, sus familias o cuidadores y las mujeres gestantes".

\section{CONCLUSIONES}

Se observa que los desafíos que se presentan en la atención integral a la primera infancia han reestructurado constantemente sus politicas hacia tres ejes fundamentales como la educacion, el cuidado y la proteccion de los menores. No obstante, para el desarrollo de las normas que se formalizan para garantizar los derechos a los niños y niñas debe actuar e incidir sobre distintos escenarios en los que el menor se desarrolla y aun más considerando las condiciones sociales y ambientales de la actual epoca teniendo encuenta factores como los contantes cambios en el medio entorno natural que incide directamente en el desarrollo fisico, biologico y por ende psicologico de los niños y niñas.

Se evidenció que el recorrido histórico sobre la educación y la atención a la primera infancia ha tenido una evolucion sobre el concepto y comprension de la infancia, desde otros contextos como el norteamericano y el europeo, en donde se han desarrolloado estrategias para orientar sobre la atencion a la primera infancia. Sin embargo, para el contexto latinoamericano aun se presentan diferencias en la comprension de la infancia y por ende se generan complicaciones a la hora de crear programas que brinden la atencion integral a los niños y las niñas. 


\section{REFERENCIAS BIBLIOGRÁFICAS}

Abeya, E.O G., Del Pino, M., Di Candia, A., Fano, V., Krupitzky, S., Fernández, M. I., \& Orazi, V. (2004). El desarrollo del niño: Una definición para la reflexión y la acción. Archivos argentinos de pediatría, 102(4), 312-313. Recuperado de http://www.sap.org.ar/organizacion/ comitesnacionales/crec_des/index.htm

Alarcón, P.LC., Castro, L.A., Schmidt, Q.M., Turriago, B.C., \& Carvajal, A.L. (Comp.). (2013). Estrategia de atención integral a la primera infancia. FUNDAMENTOS POLÍTICOS, TÉCNICOS YDE GESTIÓN. Bogotá.

Alzate, P. M. (2002). Concepciones e imagenes de la infancia. Revista de Ciencias Humanas, (28). Recuperado de https:// repository.unad.edu.co/bitstream/ handle/10596/4863/514517\%20infancia. pdf?sequence $=1$

Ancheta, A.A. (2013). La educación y atención de la primera infancia en la unión europea. Revista pedagógica, 26, 71-88. Recuperado de http://uvadoc. uva.es/bitstream/handle/10324/11993/ Tabanque-2013-26-LaEducacionYAtenci onDeLaPrimeralnfanciaEnLaUnionEu.pd f;jsessionid=2892EB6CEE05514A73AC D9154D55ABD1? sequence $=1$

Arboleda, G.V.L., \& Sierra, R.L.A. (2008). Evaluación al proceso educativo del programa FAMI —familia, mujer e infancia-en el municipio de Yarumal (Tesis de maestría). CINDE, Medellín. Recuperada de http://ridum.umanizales. edu.co:8080/xmlui/handle/6789/1586

Ardila, I. K. L., Mantilla, S. C. T., \& Bolivia, S. C. (2013). Condiciones de salud de niños menores de cinco años que participan en el programa "De cero a siempre" en Pamplona, norte de Santander. Movimiento Científico, 7(1), 6270. DOI: https://doi.org/10.33881/2011$7191 . \% 25 x$

Azorín, A. C. M. (2017). Una mirada desde los organismos internacionales a la educación para todos. Revista opción, 33 (83), 203-229. Recuperado de http://www. redalyc. org/articulo.oa?id=31053772007

Bácares, C. J. (2014). Tipologías y razones de aparición de la política pública de la infancia en Colombia 19302012. Sociedad y Economía, (26), 93120. Recuperado de http://www.scielo. org.co/pdf/soec/n26/n26a05.pdf

Bennett, J. (2011). Los sistemas de educación y cuidados en la primera infancia: Tema de tradición y gobernanza. En enciclopedia sobre el desarrollo de la primera infancia. Londres, Inglaterra.

Bernal, R., \& Camacho, A. (Comp.). (2012). La política de primera infancia en el contexto de la equidad y movilidad social en Colombia. Edición electrónica. Universidad de los Andes, Bogotá.

Blanco, G.M.R. (2005). La educación de calidad para todos empieza en la primera infancia. Revista enfoques educacionales, 7(1), 11 - 33. Recuperado de http://www.facso. uchile.cl/publicaciones/enfoques/09/ Blanco-DelPiano_N7_2005.pdf

Bodero, C.N.C. (2017). La neurociencia en la primera infancia. Apuntes de ciencia \& sociedad ,7(1),6-10. DOI: http://dx.doi. org/10.18259/acs.2017002

Bronfenbrenner, U. (1987). La ecología del desarrollo humano. Barcelona, España: Paidós 
Bronfenbrenner, U., \& Ceci, S. J. (1994). Nature-nurture reconceptualized: A bioecological model. Psychological Review, 101(4), 568-586. Recuperado de https:// psycnet.apa.org/record/1995-08473-001

Caja de herramientas para la atención integral en salud de niñas, niños y adolescentes. (2019). EncuentroAcuerdos Conceptuales y Socialización del Lineamiento para la Implementación de la Atención Integral en Salud a la Primera Infancia, Infancia y Adolescencia. Recuperado de https:// www.minsalud.gov.co/sites/rid/Lists/ BibliotecaDigital/RIDE/VS/PP/acuerdosconceptuales.pdf

Cárdenas, R.A.B., \& Gómez, D.M.C.(Coord.). (2014). Sentido de la educación inicial. DOCUMENTO NO. 20. Serie de orientaciones pedagógicas para la educación inicial en el marco de la atención integral. Bogotá: Rey Naranjo Editores.

Cathalifaud, A.M., \& Osorio, F. (1998). Introducción a los Conceptos Básicos de la Teoría General de Sistemas. Red de Revistas Científicas de América Latina, el Caribe, España y Portugal, (3), 1-12. Recuperado de Disponible en: http://www. redalyc. org/articulo.oa?id=10100306

Cordero, C.T. (2004). Educación inicial en américa latina: situaciones y retos. caso panameño. Revista Educación, 28(1): 3953. Recuperado de http://www.redalyc. org/articulo.oa?id=44028104

Decreto 4875 de 2011. Comisión Intersectorial para la Atención Integral de la Primera Infancia - AIPI - y la Comisión Especial de Seguimiento para la Atención Integral a la Primera Infancia. 22 de Diciembre de 2011. Diario Oficial No. 48.291.
Delors, J. (1996). Los cuatro pilares de la educación. Informe a la UNESCO de la Comisión internacional sobre la educación para el siglo XXI. Recuperado de http://www.unesco.org/education/pdf/ DELORS_S.PDF

Díaz, M. I., Arancibia, M., Bello, A., Salinas, L., \& Sánchez, G. (2016). Contenido, integralidad y coherencia en las políticas de primera infancia: aportes desde el currículo. Recuperado del sitio de internet http://repositorio.minedu.gob. pe/bitstream/handle/123456789/5084/ Contenido $\% 2 \mathrm{c} \% 20$ integralidad $\% 20$ y $\% 20$ coherencia $\% 20$ en $\% 20$ las $\% 20$ pol $\%$ c $3 \%$ adticas $\% 20$ de $\% 20$ primera $\% 20$ infancia $\% 20$ aportes $\% 20$ desde $\% 20$ el\% 20 curr\%c3\%adculo. pdf?sequence $=1 \&$ isAllowed=y

Documento Conpes 109 Social. (Diciembre, 2007). Política pública nacional de primera infancia "Colombia por la primera infancia”. Bogotá.

Ducoing, W.P., \& Barrón, T. C. (2017). La escuela secundariahoy: problemasy retos. Revista mexicana de investigación educativa, 22(72), 9-30. Recuperado de http://www. scielo.org. $\mathrm{mx} / \mathrm{scielo}$. php? pid=S140566662017000100009\&script=sci_arttext

Figari, C. A. S. (2009). La formación del ciudadano en la consolidación del orden civilizatorio. La concepción pedagógicapolítica de Jean-Jacques Rousseau. Perspectivas y debates en educación, 2(1),2-22. Recuperado de_https:// ri.conicet.gov.ar/handle/11336/84203

García, G.M. (1997). Educación adaptativa. Revista de Investigación Educativa, 15(2), 247-271. 
Guedeney, A., \& Pérez, C. M. (2015). Retraimiento social en la primera infancia. Revista uruguaya de Psicoanálisis, 120, 120-132.Recuperado de https://www.apuruguay.org/ apurevista/2010/16887247201512008. pdf

ICBF. (2006). COLOMBIA POR LA PRIMERA INFANCIA. Política pública por los niños y niñas, desde la gestación hasta los 6 años. Bogotá, Colombia.

ICBF. (2017). Manual operativo. MODALIDAD FAMILIAR PARA LA ATENCIÓN A LA PRIMERA INFANCIA. Bogotá: Sistema Colombiano de Bienestar Familiar

ICBF. (2018). LINEAMIENTO TÉCNICO PARA LA ATENCIÓN A LA PRIMERA INFANCIA. Bogotá: Sistema Colombiano de Bienestar Familiar.

Izzedin, B. R., \& Pachajoa L. A. (2009). Pautas, prácticas y creencias acerca de crianza... ayer y hoy. Liberabit, 15(2), 109-115. Recuperado de http://www.scielo.org.pe/ scielo.php?pid=S1729482720090002000 05\&script=sci_arttext

Jaramillo, L. (2007). Concepciónes de infancia. Zona próxima, (8), 16572416. Recuperado de http://rcientificas. uninorte.edu.co/index.php/zona/article/ viewArticle/1687/4634

Ley 1098 de 2006. Código de la Infancia y la Adolescencia.8 de Noviembre de 2006. Diario Oficial No. 46.446.

Ley 1295 de 2009. La atención integral de los niños y las niñas de la primera infancia de los sectores clasificados como 1, 2 y 3 del Sisbén. 6 de Abril de 2009. Diario Oficial No. 47.314 .

Lombardi, J. (2004). Desarrollo de la Primera.
Trabajo presentado en el II Simposio Inter Americano: Políticas y Estrategias para unatransición exitosa de los niños hacia la Socialización y la Escuela. Valparaíso, Chile, 27 - 29 de Mayo.

Lynn, S. K., Caridad, M. A., Jaimovich, A., \& Cruz-Aguayo, Y. (2016). Una mirada al desarrollo infantil en América Latina y el Caribe desde la teoría y el pensamiento sistémico. Washington D. C., Estados Unidos: BID.

Midaglia, C., Barba, G. M. O., \& Lomelí, E. V. (Eds.). (2018). Políticas sociales en América Latina en los inicios del siglo $X X I$ : innovaciones, inercias y retrocesos. El Colegio de la Frontera Norte.

Ministerio de Educación Nacional. (2010). Guía operativa para la prestación del servicio de atención integral a la primera infancia. Colombia: Oficina de comunicaciones del Ministerio de Educación Nacional.

Myers, R. G. (2000). Atención y desarrollo de la primera infancia en Latinoamérica y El Caribe: Una revisión de los diez últimos años y una mirada hacia el futuro. Revista Iberoamericana de educación, 22(1), 17-39. Recuperado de https:// inversionenlainfancia.net/application/ views/materiales/UPLOAD/ARCHIVOS_ DOCUMENTO/documento_documento_ file/21_02\%20Atencion $\% 20$ y 20 desarrollo\%20de \%20la\%20primera $\% 20$ infancia\%20-\%20Myers\%2099.pdf

Narodowski, M., \& Martínez Boom, A. (2016). ¿Por qué se expande la educación privada?: Aportes para el debate global. Revista Colombiana de Educación, (70), 17-26. Recuperado de http://www.scielo. org.co/pdf/rcde/n70/n70a02.pdf 
Pachón, X. (2007). La familia en Colombia a lo largo del siglo XX. Familias, cambios $y$ estrategias, 1-16. Recuperado de http://www.bdigital.unal.edu. co/1363/13/12CAPI11.pdf

Paya, R. A. (2010). Políticas de educación inclusiva en América Latina Propuestas, realidades y retos de futuro. Revista de educación inclusiva, 3(2), 125142. Recuperado de https://www. revistaeducacioninclusiva.es/index.php/ REl/article/view/209/203

Peralta, E.M.V., \& Fujimoto,G.G. (ed.). (1998). La atención integral de la primera infancia en américa latina: ejes centrales y los desafíos para el siglo XXI. Organización De Estados Americanos (O.E.A). Santiago de Chile.

Pineda, E. O. M., \& Orozco, A. P. P. (2018). Estado del arte y abordaje del concepto de ludificación en el aprendizaje en primera infancia. Infancias imágenes,17(2), 147162. DOI: $10.14483 / 16579089.12397$

Quintero, A. S.R., Ramírez, R. L.E., \& Jaramillo, V.B. (2016). Actitud lúdica y lenguajes expresivos en la educación de la primera infancia. Revista Virtual Universidad Católica del Norte, 48, 155-170. Recuperado de http://revistavirtual.ucn. edu.co/index.php/RevistaUCN/article/ view/766/1292

Rincón, J. (2019). Exclusión social de la infancia afrocolombiana en el aula escolar desde un enfoque bioético. Revista Colombiana de Educación, 76, 305-320. doi:10.17227/ rce.num76-9384

Rodríguez E. C. M., \& Marzonetto, G. L. (2015). El trabajo de cuidado remunerado: Estudio de las condiciones de empleo en la educación básica y en el trabajo en casas particulares. CONICET, 4 (4), 1-50.
Recuperado de https://ri.conicet.gov.ar/ handle/11336/56796

Rodríguez, I. P. (2000). ¿Sociología de la Infancia? Aproximaciones a un campo de estudio difuso. Revista Internacional de Sociología, 58(26), 99-124. DOI: https://doi.org/10.3989/ris.2000.i26.796

Rozo-Gutiérrez, N., \& Vargas-Trujillo, Z. (2018). Análisis de Política Pública de Infancia y Adolescencia, 2011-2021, en Bogotá, DC, Colombia. Desafíos, 30(2), 279-314. DOI: http://dx.doi.org/10.12804/revistas. urosario.edu.co/desafios/a.5325

Ruiz, G. (2013). La teoría de la experiencia de John Dewey: significación histórica y vigencia en el debate teórico contemporáneo. Foro de Educación, 11(15), 103-124. DOI: http://dx.doi. org/10.14516/fde.2013.011.015.005

Salinas-Quiroz, F., Morales-Carmona, F. A., de Castro, F., Juárez-Hernández, M. C., Posada, G., \& Carbonell, O. A. (2015). Educación Inicial de Base Segura: Indicador de la calidad educativa para la primera infancia. Psicología Iberoamericana, 23(1), 75-82. Recuperado de http://www.redalyc.org/ articulo.oa?id=133944230009

Sánchez, T. L., Murcia, L. O., Trujillo, M., \& Pérez, T. H. (2016). Las políticas públicas de orden nacional y distrital de primera infancia, una mirada desde el enfoque de capacidades. Revista Interamericana de Investigación, Educación y Pedagogía, RIIEP, 9(1). DOI: https://doi. org/10.15332/25005421

Serrano-Arenas, D., Ochoa-Cervantes, A., \& Arcos-Miranda, E. (2019). Conceptualizaciones, perspectivas y referentes de la participación en la educación primaria, México. Revista 
Latinoamericana de Ciencias

Sociales, Niñez y Juventud, 17(2), 35-57. Recuperado de http://www. scielo.org.co/scielo.php? pid=S1692$715 \times 2019000200035 \&$ script $=s c i$ abstract\&tlng=en

Torres, R.H.Y., \& Ramos, V. V. (2016). Influencia de los medios en el desarrollo integral del niño en la primera infancia. Praxis Investigativa ReDIE,8(14), 210-218. Recuperado de https://dialnet.unirioja.es/ servlet/articulo?codigo $=6556776$

Tuñón, I. (2015). (Coord.). Desafíos del desarrollo humano en la primera infancia. ( $1^{\mathrm{a}}$ ed.). Ciudad Autónoma de Buenos Aires: Biblos.

UNESCO. (2000). Marco de Acción de Dakar. Educación para Todos: cumplir nuestros compromisos comunes. Foro Mundial sobre la Educación.

UNESCO. (2007). Educación de calidad para todos: un asunto de derechos humanos. Buenos Aires, Argentina.

UNICEF (2006). Convención Internacional de los derechos del Niño. Recuperado del sitio de internet https://www.un.org/es/ events/childrenday/pdf/derechos.pdf

UNICEF. (2005). Estado Mundial de la Infancia 2005: La infancia amenazada (informe). Recuperado del sitio de internet UNICEF https://www.unicef.org/spanish/sowc05/ sowc05_sp.pdf

UNICEF. (2014). La inversión en la primera infancia en América latina. Propuesta metodológica y análisis en países seleccionados de la región. Recuperado de http://www.sipi.siteal.iipe.unesco. org/publicaciones/1186/la-inversion-enla-primera-infancia-en-america-latinapropuesta-metodologica-y
Universidad de Costa Rica. (2015). Estado de los Derechos de la Niñez y Adolescencia. San José, Costa Rica.

Vaillant, E.D. (2019). Formación Inicial del Profesorado de Educación Secundaria en América Latina- Dilemas y Desafíos. Revista de Currículum y formación del profesorado,23(3). Recuperado de https://revistaseug.ugr.es/index.php/ profesorado/article/view/9516

Viciana, V. G., Cano, L. G., Chacón, R.C., Padial, R.R., \& Martínez, M.A. (2017). Importancia de la motricidad para el desarrollo integral del niño en la etapa de educación infantil. EmásF: revista digital de educación física, (47), 89-105. Recuperado de https://dialnet.unirioja.es/ servlet/articulo?codigo $=6038088$

Vigotsky, L. (1979). Interacción entre aprendizaje y desarrollo en el desarrollo de los procesos psicológicos superiores. 123-140. España: Critica-Grijalbo.

Vilafranca, I. M. (2012). La filosofía de la educación de Rousseau: el naturalismo eudamonista. Educació i Història: revista d'història de l'educació, 19, 35-53. DOI: 10.2436/20.3009.01.94

Zapata-Ospina, B. E. \& Restrepo-Mesa, J. H. (2013). Aprendizajes relevantes para los niños y las niñas en la primera infancia. Revista Latinoamericana de Ciencias Sociales, Niñez y Juventud, 11 (1), 217-227. Recuperado de http:// revistaumanizales.cinde.org.co/rlcsnj/ index.php/Revista-Latinoamericana/ article/view/847/416 